Environmental Applied Science and Management

Jan 2015

\title{
Invasive Aquatic Plants in the Aquarium and Ornamental Pond Industries
}

While enrolled in Ryerson University's Environmental Applied Science and Management MASc program, Shakira Azan undertook her thesis research on the potential for invasive plant species to be introduced to southern Ontario waterways through the aquarium and ornamental pond industries. With her supervisors Drs. Andy Laursen and Michal Bardecki, Shakira co-authored a paper that details her findings and is soon to be published in the journal Weed Research.

\section{Alien Invasions}

In their paper, Shakira and her co-authors offer an assessment of the invasive risk associated with the establishment and dispersal of plants available in the aquarium and ornamental pond industries in the Greater Toronto area. "The ability to clarify and recognize the common traits of a successful alien-plant invaders," Shakira explains, "is absolutely essential for helping to prevent future invasions."

Development of the risk model Shakira used in her research required assessing "pathway risk potential. In order to calculate this, she used actual sales volumes of individual taxa as a proxy for propagule pressure (i.e., the number of non-native species released into an area). One of the most notable aspects of Shakira's study was her identification of 642 taxa (taxonomic groupings such as genus, species and family) for sale; that was a far higher number than other inventories of aquatic plants for sale by aquarium and ornamental pond retailers elsewhere.

\section{Organism Risk Potential}

Organism risk potential is the ability to become established and disperse associated with a release. In order to establish this potential, Shakira - who is now a PhD student in the Department of Biology at Queen's University conducted a comprehensive analysis of each species' biological traits, employing a discriminant correspondence analysis to predict which biological traits were useful in discriminating invasive plants and non-invasive plant taxa. "Importantly," says Shakira, "I found that a relatively small number of biological traits were useful in predicting whether an alien aquatic plant had the characteristics that would support establishment and/or dispersal in new environments."

\section{High-Risk Species}

Shakira's research led her to conclude that the aquatic plants that are most concerning as potential invaders are ones that are cold tolerant, able to propagate by fragments and use a number of different dispersal methods. Her model identified 11 alien plants (see Figure 1) in the trade that have a high risk of becoming invasive, and an additional 52 with moderately high risk.

\section{Funding}

Financial support for Shakira's research came from Ryerson's programs in Environmental Applied Science and Management, as well as from the Department of Chemistry and Biology. 